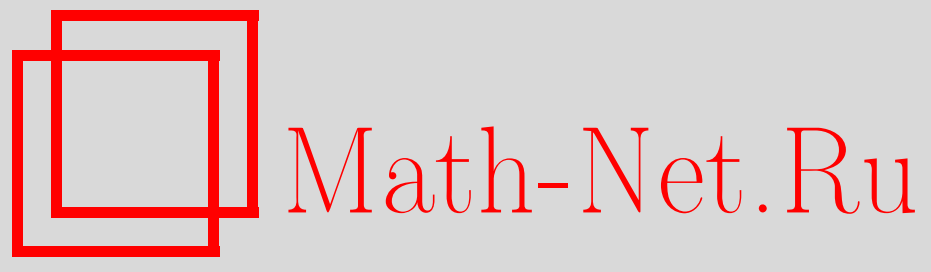

М. В. Шамолин, Новый случай интегрируемости уравнений динамики на касательном расслоении к трехмерной сфере, УМН, 2013, том 68, выпуск 5, 185-186

DOI: https://doi.org/10.4213/rm9541

Использование Общероссийского математического портала Math-Net.Ru подразумевает, что вы прочитали и согласны с пользовательским соглашением http://www . mathnet.ru/rus/agreement

Параметры загрузки:

IP : 52.90 .164 .192

26 апреля 2023 г., 18:29:43

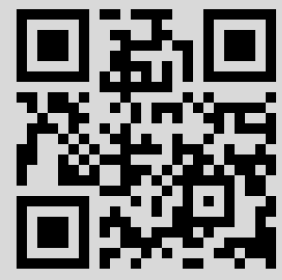




\section{Новый случай интегрируемости уравнений динамики на касательном расслоении к трехмерной сфере}

\section{М. В. Шамолин}

Пусть четырехмерное твердое тело $\Theta$ массы $m$ с гладкой трехмерной границей $\partial \Theta$ имеет в некоторой связанной с ним системе координат $D x_{1} x_{2} x_{3} x_{4}$ тензор инерции вида $\operatorname{diag}\left\{I_{1}, I_{2}, I_{2}, I_{2}\right\}$. Расстояние от точки $N$ приложения силы $\mathbf{S}$ до точки $D$ является функцией, по крайней мере, некоторого угла $\alpha: D N=R(\alpha, \ldots)$ (ср. с [1]-[3]). Сила $\mathbf{S}$ имеет величину $S=s(\alpha) \operatorname{sgn} \cos \alpha \cdot v^{2},\left|\mathbf{v}_{D}\right|=v$, где $s-$ некоторая функция, характеризующая в системе как рассеяние, так и подкачку энергии [1], [2]. При этом $S=S_{v}(\alpha)=B v^{2} \cos \alpha, B>0$.

Если $\Omega$ - тензор угловой скорости тела $\Theta, \Omega \in \mathrm{so}(4)$, то часть уравнений движения, отвечающая алгебре Ли so(4), имеет следующий вид [2]:

$$
\dot{\Omega} \Lambda+\Lambda \dot{\Omega}+[\Omega, \Omega \Lambda+\Lambda \Omega]=M
$$

где $\Lambda=\operatorname{diag}\left\{\lambda_{1}, \lambda_{2}, \lambda_{3}, \lambda_{4}\right\}, \lambda_{1}=\left(-I_{1}+I_{2}+I_{3}+I_{4}\right) / 2, \ldots, \lambda_{4}=\left(I_{1}+I_{2}+I_{3}-I_{4}\right) / 2$, $M$ - момент внешних сил, действующих на тело в $\mathbb{R}^{4}$, спроектированный на естественные координаты в алгебре Ли so(4), $[\cdot, \cdot]$ - коммутатор в so(4). Кососимметрическая матрица $\Omega \in \mathrm{so}(4)$ определяется величинами $\omega_{1}, \omega_{2}, \omega_{3}, \omega_{4}, \omega_{5}, \omega_{6}$ - компонентами в алгебре Ли so(4). При этом, очевидно, $\lambda_{i}-\lambda_{j}=I_{j}-I_{i}$ для любых $i, j=1, \ldots, 4$.

При вычислении момента внешней силы строится отображение $\mathbb{R}^{4} \times \mathbb{R}^{4} \rightarrow \operatorname{so}(4)$, переводящее пару векторов из $\mathbb{R}^{4}$ в некоторый элемент из алгебры Ли so(4) [2], [3].

Уравнение Ньютона движения центра масс $C$ тела $\Theta$ представится в виде $m \mathbf{w}_{C}=\mathbf{F}$, где по многомерной формуле Ривальса $\mathbf{w}_{C}=\mathbf{w}_{D}+\Omega^{2} \mathbf{D C}+E \mathbf{D C}, \mathbf{w}_{D}=\dot{\mathbf{v}}_{D}+\Omega \mathbf{v}_{D}$, $E=\dot{\Omega}, \mathbf{F}-$ внешняя сила, действующая на тело (в нашем случае $\mathbf{F}=\mathbf{S}$ ), $E$ - тензор углового ускорения [1], [2].

Несколько расширим задачу: по прямой $D x_{1}=D C$ будет действовать некоторая следящая сила Т. В данной работе разобран такой класс движения, когда центр масс тела движется прямолинейно и равномерно (т. е. $\mathbf{V}_{C} \equiv$ const).

Если $N=\left(0, x_{2 N}, x_{3 N}, x_{4 N}\right)$ в системе $D x_{1} x_{2} x_{3} x_{4}, \mathbf{S}=\{-S, 0,0,0\}$, то момент силы в координатах алгебры Ли so(4) имеет следующий вид: $\left\{0,0, x_{4 N} S, 0,-x_{3 N} S, x_{2 N} S\right\} \in$ $\mathbb{R}^{6} \cong M \in \operatorname{so}(4)$.

При этом, если $\left(v, \alpha, \beta_{1}, \beta_{2}\right)$ - сферические координаты в $\mathbb{R}^{4}$, то $x_{2 N}=A \sin \alpha \cos \beta_{1}-$ $h \omega_{6} / v, x_{3 N}=A \sin \alpha \sin \beta_{1} \cos \beta_{2}+h \omega_{5} / v, x_{4 N}=A \sin \alpha \sin \beta_{1} \sin \beta_{2}-h \omega_{3} / v, A, h>0$.

Очевидно, что уравнения (1) существуют три циклических первых интеграла: $\omega_{1}=$ $\omega_{1}^{0}, \omega_{2}=\omega_{2}^{0}, \omega_{4}=\omega_{4}^{0}$, при этом считаем, что $\omega_{1}^{0}=\omega_{2}^{0}=\omega_{4}^{0}=0$. В результате оставшиеся уравнения на so(4) примут вид $\dot{\omega}_{3}=n_{0}^{2} v^{2} \sin \alpha \cos \alpha \sin \beta_{1} \sin \beta_{2}-h B \omega_{3} v \cos \alpha /\left(2 I_{2}\right)$, $\dot{\omega}_{5}=-n_{0}^{2} v^{2} \sin \alpha \cos \alpha \sin \beta_{1} \cos \beta_{2}-h B \omega_{5} v \cos \alpha /\left(2 I_{2}\right), \quad \dot{\omega}_{6}=n_{0}^{2} v^{2} \sin \alpha \cos \alpha \cos \beta_{1}-$ $h B \omega_{6} v \cos \alpha /\left(2 I_{2}\right)$.

Тогда замена угловых скоростей $z_{1}=\omega_{3} \cos \beta_{2}+\omega_{5} \sin \beta_{2}, z_{2}=-\omega_{3} \sin \beta_{2} \cos \beta_{1}+$ $\omega_{5} \cos \beta_{2} \cos \beta_{1}+\omega_{6} \sin \beta_{1}, z_{3}=\omega_{3} \sin \beta_{2} \sin \beta_{1}-\omega_{5} \cos \beta_{2} \sin \beta_{1}+\omega_{6} \cos \beta_{1}$ приводит совместную систему из уравнения Ньютона и (1) к следующему виду: $(\sigma=D C$, $\left.b=\sigma n_{0}, H_{1}=h B /\left(2 I_{2} n_{0}\right),[b]=\left[H_{1}\right]=1, n_{0}^{2}=A B /\left(2 I_{2}\right)\right)$ :

$$
\dot{v}=\sigma \cos \alpha\left[n_{0}^{2} v^{2} \sin ^{2} \alpha-\left(z_{1}^{2}+z_{2}^{2}+z_{3}^{3}\right)\right]-b H_{1} v z_{3} \sin \alpha \cos \alpha
$$

Работа выполнена при поддержке РФФИ (грант № 12-01-00020-а).

DOI: $10.4213 / \mathrm{rm} 9541$ 


$$
\left.\begin{array}{r}
\dot{\alpha}=-z_{3}+\sigma n_{0}^{2} v \sin \alpha \cos ^{2} \alpha+\sigma \sin \alpha\left(z_{1}^{2}+z_{2}^{2}+z_{3}^{3}\right) / v-b H_{1} z_{3} \cos ^{2} \alpha, \\
\dot{z}_{3}=n_{0}^{2} v^{2} \sin \alpha \cos \alpha-\left(1+b H_{1}\right)\left(z_{1}^{2}+z_{2}^{2}\right) \operatorname{ctg} \alpha-H_{1} v z_{3} \cos \alpha, \\
\dot{z_{2}}=\left(1+b H_{1}\right) z_{2} z_{3} \operatorname{ctg} \alpha+\left(1+b H_{1}\right) z_{1}^{2} \operatorname{ctg} \alpha \operatorname{ctg} \beta_{1}-H_{1} v z_{2} \cos \alpha, \\
\dot{z_{1}}=\left(1+b H_{1}\right) z_{1} z_{3} \operatorname{ctg} \alpha-\left(1+b H_{1}\right) z_{1} z_{2} \operatorname{ctg} \alpha \operatorname{ctg} \beta_{1}-H_{1} v z_{1} \cos \alpha, \\
\dot{\beta}_{1}=\left(1+b H_{1}\right) z_{2} \operatorname{ctg} \alpha,
\end{array}\right\}
$$

В системе седьмого порядка (2)-(4) появилась независимая подсистема шестого порядка (2), (3). Для полного интегрирования нужно искать, вообще говоря, шесть независимых первых интегралов. Однако после замен $z=\sqrt{z_{1}^{2}+z_{2}^{2}}, z_{*}=z_{2} / z_{1}$, $z=n_{0} v Z, z_{k}=n_{0} v Z_{k}, k=1,2,3, z_{*}=Z_{*},\langle\cdot\rangle \mapsto n_{0} v\left\langle{ }^{\prime}\right\rangle$ она приводится к виду

$$
\begin{aligned}
& v^{\prime}=v \Psi\left(\alpha, Z, Z_{3}\right), \quad \Psi\left(\alpha, Z, Z_{3}\right)=b \cos \alpha\left[\sin ^{2} \alpha-\left(Z^{2}+Z_{3}^{2}\right)\right]-b H_{1} Z_{3} \sin \alpha \cos \alpha, \\
& \alpha^{\prime}=-Z_{3}+b \sin \alpha \cos ^{2} \alpha+b \sin \alpha\left(Z^{2}+Z_{3}^{2}\right)-b H_{1} Z_{3} \cos ^{2} \alpha, \\
& \left.\begin{array}{r}
Z_{3}^{\prime}=\sin \alpha \cos \alpha-\left(1+b H_{1}\right) Z^{2} \operatorname{ctg} \alpha-Z_{3} \Psi\left(\alpha, Z, Z_{3}\right)-H_{1} Z_{3} \cos \alpha, \\
Z^{\prime}=\left(1+b H_{1}\right) Z Z_{3} \operatorname{ctg} \alpha-Z \Psi\left(\alpha, Z, Z_{3}\right)-H_{1} Z \cos \alpha,
\end{array}\right\} \\
& Z_{*}^{\prime}= \pm\left(1+b H_{1}\right) Z \sqrt{1+Z_{*}^{2}} \operatorname{ctg} \alpha \operatorname{ctg} \beta_{1}, \quad \beta_{1}^{\prime}= \pm\left(1+b H_{1}\right) \frac{Z Z_{*}}{\sqrt{1+Z_{*}^{2}}} \operatorname{ctg} \alpha, \\
& \beta_{2}^{\prime}=\mp\left(1+b H_{1}\right) \frac{Z_{1}}{\sqrt{1+Z_{*}^{2}}} \operatorname{ctg} \alpha \csc \beta_{1},
\end{aligned}
$$

при этом систему (7)-(8) можно рассматривать на касательном расслоении $T \mathbb{S}^{3}$ трехмерной сферы.

Видно, что система пятого порядка (3) распалась на независимые подсистемы более низкого порядка: система (6) - третьего, а система (7) (после замены независимого переменного) - второго. Поэтому для полной интегрируемости достаточно указать два независимых интеграла системы (6), один - системы (7) и два дополнительных интеграла, "привязывающих" уравнения (5) и (8).

Полная система седьмого порядка обладает аналитическим первым интегралом вида $v^{2}\left(1-2 b Z_{3} \sin \alpha+b^{2}\left(Z^{2}+Z_{3}^{2}\right)\right)=V_{C}^{2}$.

Система (6) обладает двумя независимыми первыми интегралами, являющимися трансцендентными функциями фазовых переменных (в смысле комплексного анализа) и выражающимися через конечную комбинацию элементарных функций (ср. [1]-[3]):

$$
\frac{Z^{2}+Z_{3}^{2}-b Z_{3} \sin \alpha+\sin ^{2} \alpha}{Z \sin \alpha}=C_{1}=\text { const, } \quad G\left(\frac{Z}{\sin \alpha}, \frac{Z_{3}}{\sin \alpha}, \sin \alpha\right)=C_{2}=\text { const. }
$$

Система (7) имеет первый интеграл вида $\sqrt{1+Z_{*}^{2}} / \sin \beta_{1}=C_{3}=$ const, и, в свою очередь, дополнительный первый интеграл, позволяющий определить величину $\beta_{2}$, имеет вид $\pm \cos \beta_{1} / \sqrt{C_{3}^{2}-1}=\sin \left\{C_{3}\left(\beta_{2}+C_{4}\right)\right\}, C_{4}=$ const.

\section{Список литературы}

[1] М. В. Шамолин, Методы анализа динамических систем с переменной диссипацией в динамике твердого тела, Изд-во "Экзамен”, М., 2007, 352 с. [2] М. В. Шамолин, Докл. PAH, 375:3 (2000), 343-346. [3] М. В. Шамолин, УМН, 53:3(321) (1998), 209-210.

M. В. Шамолин (M. V. Shamolin)

Московский государственный университет им. М. В. Ломоносова

E-mail: shamolin@imec.msu.ru, shamolin@rambler.ru
Представлено А. В. Михалёвым Принято редколлегией 05.08 .2013 